\title{
ON THE LIMITED $p$-SCHUR PROPERTY OF SOME OPERATOR SPACES
}

\author{
M.B. DEHGHANI ${ }^{1}$, S.M. MOSHTAGHIOUN ${ }^{1, *}$ AND M. DEHGHANI ${ }^{2}$ \\ ${ }^{1}$ Department of Mathematics, yazd University, P. O. Box 89195-741, Yazd, Iran \\ ${ }^{2}$ Department of Pure Mathematics, Faculty of Mathematical Sciences, University of Kashan, P. O. Box \\ 87317-53153, Kashan, Iran \\ ${ }^{*}$ Corresponding author: moshtagh@yazd.ac.ir
}

\begin{abstract}
We introduce and study the notion of limited $p$-Schur property $(1 \leq p \leq \infty)$ of Banach spaces. Also, we establish some necessary and sufficient conditions under which some operator spaces have the limited $p$-Schur property. In particular, we prove that if $X$ and $Y$ are two Banach spaces such that $X$ contains no copy of $\ell_{1}$ and $Y$ has the limited $p$-Schur property, then $K(X, Y)$ (the space of all compact operators from $X$ into $Y$ ) has the limited $p$-Schur property.
\end{abstract}

\section{INTRODUCTION}

A non-empty subset $K$ of a Banach space $X$ is said to be limited (resp Dunford-Pettis (DP)), if for every weak* ${ }^{*}$-null (resp. weakly null) sequence $\left(x_{n}^{*}\right)$ in the dual space $X^{*}$ of $X$ converges uniformly on $K$, that is,

$$
\lim _{n \rightarrow \infty} \sup _{x \in K}\left|\left\langle x, x_{n}^{*}\right\rangle\right|=0
$$

where $\left\langle x, x^{*}\right\rangle$ denotes the duality between $x \in X$ and $x^{*} \in X^{*}$. In particular, a sequence $\left(x_{n}\right) \subset X$ is limited if and only if $\left\langle x_{n}, x_{n}^{*}\right\rangle \rightarrow 0$, for all weak*-null sequences $\left(x_{n}^{*}\right)$ in $X^{*}$.

Received $11^{\text {th }}$ September, 2017; accepted $27^{\text {th }}$ November, 2017; published $3^{\text {rd }}$ January, 2018.

2010 Mathematics Subject Classification. 47L05; 46B25.

Key words and phrases. Schur property; $p$-Schur property; limited $p$-Schur property; limited $p$-converging; weakly $p$-compact. (C)2018 Authors retain the copyrights of their papers, and all open access articles are distributed under the terms of the Creative Commons Attribution License. 
A subset $K$ of a Banach space $X$ is a limited set if and only if for any Banach space $Y$, every pointwise convergent sequence $\left(T_{n}\right) \subset L(X, Y)$ converges uniformly on $K$, where $L(X, Y)$ denoted the space of all bounded operators from $X$ into $Y$ [17, Corollary 1.1.2].

It is easily seen that every relatively compact subset of a Banach space is limited. But the converse is not true, in general. If every limited subset of Banach space $X$ is relatively compact, then $X$ has the Gelfand-Phillips $(G P)$ property. For example, the classical Banach space $c_{0}$ and $\ell_{1}$ have the GP property and every reflexive space and dual space containing no copy of $\ell_{1}$ have the same property.

A sequence $\left(x_{n}\right)$ in Banach space $X$ is called weakly $p$-summable with $1 \leq p<\infty$, if for each $x^{*} \in X^{*}$, the sequence $\left(\left\langle x_{n}, x^{*}\right\rangle\right) \in \ell_{p}$ and a sequence $\left(x_{n}\right)$ in $X$ is said to be weakly $p$-convergent to $x \in X$ if the sequence $\left(x_{n}-x\right) \in \ell_{p}^{w e a k}(X)$, where $\ell_{p}^{\text {weak }}(X)$ denoted the space of all weakly $p$-summable sequence in $X$. Also a bounded set $K$ in a Banach space is said to be relatively weakly $p$-compact, $1 \leq p \leq \infty$ if every sequence in $K$ has a weakly $p$-convergent subsequence. If the limit point of each weakly $p$-convergent subsequence is in $K$, then we call $K$ weakly $p$-compact set. Also, a Banach space $X$ is weakly $p$-compact if the closed unit ball $B_{X}$ of $X$ is a weakly $p$-compact set. An operator $T \in L(X, Y)$ is said to be $p$-converging if it transfers weakly $p$-summable sequence into norm null sequences. The class of all $p$-converging operators from $X$ into $Y$ is denoted by $C_{p}(X, Y)$.

An operator $T \in L(X, Y)$ is limited $p$-converging if it transfers limited and weakly $p$-summable sequences into norm null sequences. we denote the space of all limited $p$-converging operators from $X$ into $Y$ by $C_{l p}(X, Y)[7]$.

A Banach space $X$ has the Schur property if every weakly null sequence in $X$ converges in norm. The simplest Banach space with the Schur property is $\ell_{1}$. Also a banach space $X$ has the $p$-Schur property $(1 \leq p \leq \infty)$ if every weakly $p$-summable subset of $X$ is compact. In other words, if $1 \leq p<\infty, X$ has the $p$-Schur property if and only if every sequence $\left(x_{n}\right) \in \ell_{p}^{\text {weak }}(X)$ is a norm null sequence, for example, $\ell_{p}$ has the 1-Schur property. Moreover, $X$ has the $\infty$-Schur property if and only if every sequence in $c_{0}^{\text {weak }}(X)$ in norm null where $c_{0}^{\text {weak }}(X)$ containing all weakly null sequences in $X$. So $\infty$-Schur property coincides with the Schur property. Also one note that every Schur space has the $p$-Schur property [6].

The reader is referred to $[2,11,14-16]$ for more information about these concepts.

In this note, we study the limited $p$-Schur property of some operator spaces, specially, the space of compact operators. We prove that if $X$ and $Y$ are two Banach spaces such that $X$ contains no copy of $\ell_{1}$ and $Y$ has the limited $p$-Schur property, then $K(X, Y)$ has the limited $p$-Schur property. Finally, we conclude that if $\left(X_{\alpha}\right)_{\alpha \in I}$ are Banach spaces and $X=\left(\oplus_{\alpha \in I} X_{\alpha}\right)_{1}$ their $\ell_{1}$-sum, then the space $X$ has the $p$-Schur property if and only if each factor $X_{\alpha}$ has the same property. 


\section{MAin RESUlts}

Recall that the Banach space $X$ has the limited $p$-Schur property if every limited weakly $p$-compact subset of $X$ is relatively compact. More precisely, the Banach space $X$ has the limited $p$-Schur property if and only if every limited sequence $\left(x_{n}\right) \in \ell_{p}^{w e a k}(X)$ is norm null. It is easy to see that every Banach space with the $p$-Schur property and every Banach space with GP property is limited $p$-Schur [7]. Moreover, a Banach space $X$ has the GP property if and only if every limited weakly null sequence in $X$ is norm null [2, Proposition 6.8]. Therefore the limited Schur (i.e., limited $\infty$-Schur ) property is equivalent to the GP property. Also, if a Banach space $X$ have the limited $p$-Schur and $D P_{p}^{*}$ properties, then it has the $p$-Schur property. Indeed, a Banach space $X$ is said to have the $D P^{*}$-property of order $p\left(D P_{p}^{*}\right)$ if all weakly $p$-compact sets in $X$ are limited [10].

Recall that if $M$ is a closed subspace of $L(X, Y)$, then for arbitrary elements $x \in X$ and $y^{*} \in Y^{*}$, the evaluation operators $\phi_{x}: M \rightarrow Y$ and $\psi_{y^{*}}: M \rightarrow X^{*}$ on $M$ are defined by

$$
\phi_{x}(T)=T x, \quad \psi_{y^{*}}(T)=T^{*} y^{*}, \quad(T \in M)
$$

Theorem 2.1. Let $X$ and $Y$ be two Banach spaces such that $X$ is weakly $p$-compact and $Y$ has the $p$-Schur property. Then $L(X, Y)$ has the limited p-Schur property.

Proof. Suppose that $\left(T_{n}\right)$ is a limited weakly $p$-summable sequence in $L(X, Y)$. We have to prove that $\left(T_{n}\right)$ is norm null. We first observe that for every $x \in X$ the evaluation operator $\phi_{x}$ from $L(X, Y)$ to $Y$ maps the sequence $\left(T_{n}\right)$ to the sequence $\left(T_{n} x\right)$. So the latter is also a limited weakly $p$-summable sequence in $Y$. Therefore $\left\|T_{n} x\right\| \rightarrow 0$, since $Y$ has the limited $p$-Schur property.

Now, suppose that $\left(T_{n}\right)$ is not norm null. Then there is a sequence $\left(x_{n}\right)$ in $X$ and $\varepsilon>0$ such that

$$
\left\|T_{n} x_{n}\right\|>2 \varepsilon
$$

for all $n \in \mathbb{N}$. Since $X$ is weakly $p$-compact we may assume that there exists $x \in X$ such that $\left(x_{n}-x\right) \in$ $\ell_{p}^{w e a k}(X)$. As $\left\|T_{n} x\right\| \rightarrow 0$, we may finally suppose that $f_{n}=\left\|T_{n} x_{n}-T_{n} x\right\|>\varepsilon$ for all $n \in \mathbb{N}$. Now, choose functional $y_{n}^{*}$ in $B_{Y^{*}}$ so that $\left\langle T_{n} x_{n}-T_{n} x, y_{n}^{*}\right\rangle=f_{n}$, and define $\Lambda_{n} \in L(X, Y)^{*}$ by

$$
\left\langle T, \Lambda_{n}\right\rangle=\left\langle T x_{n}-T x, y_{n}^{*}\right\rangle
$$

for all $T \in L(X, Y)$. Since $\left|\left\langle T, \Lambda_{n}\right\rangle\right| \leq\left\|T x_{n}-T x\right\| \rightarrow 0$, because $\left(x_{n}-x\right) \in \ell_{p}^{w e a k}(X)$, we see that $\left(\Lambda_{n}\right)$ is a weak*-null sequence. But $\left\langle T_{n}, \Lambda_{n}\right\rangle=f_{n}>\varepsilon>0$ for all $n \in \mathbb{N}$. Contradicting the assumption that $\left(T_{n}\right)$ is limited.

Corollary 2.1. Let $X$ and $Y$ be two Banach spaces. If $X$ is reflexive and $Y$ has the Schur property, then $L(X, Y)$ has the GP property. 
Proof. Let $p=\infty$ in Theorem 2.1 .

Corollary 2.2. Let $X$ and $Y$ be two Banach spaces. If $X$ is a weakly p-compact and $Y^{*}$ has the p-Schur property, then $\left(X \widehat{\otimes}_{\pi} Y\right)^{*}$ has the limited p-Schur property.

Proof. It follows easily from the fact that $L\left(X, Y^{*}\right)=\left(X \widehat{\otimes}_{\pi} Y\right)^{*}$.

Corollary 2.3. Let $X$ and $Y$ be two Banach spaces. If $X^{*}$ has the p-Schur property and $Y^{*}$ is weakly p-compact, then $L(X, Y)$ has the limited p-Schur property.

Proof. The mapping $T \mapsto T^{*}$ maps $L(X, Y)$ onto a closed subspace of $L\left(Y^{*}, X^{*}\right)$, which has the limited p-Schur property by virtue of Theorem 2.1.

In the following theorem we give a necessary and sufficient condition for which a Banach space has the limited $p$-Schur property.

Theorem 2.2. The Banach space $X$ has the limited $p$-Schur property if and only if $L(X, Y)=C_{l p}(X, Y)$, for every Banach space $Y$.

Proof. Suppose that $X$ has the limited $p$-Schur property. If $T \in L(X, Y)$ and $\left(x_{n}\right) \in \ell_{p}^{w e a k}(X)$ is a limited sequence, then $\left\|x_{n}\right\| \rightarrow 0$. Hence $\left\|T x_{n}\right\| \rightarrow 0$.

Conversely, If $Y=X$, then the identity operator on $X$ is belongs to $C_{l p}$. Therefore $X$ has the limited p-Scuhr property.

Similarly, we can prove that the Banach space $X$ has the limited $p$-Schur property if and only if $L(Y, X)=$ $C_{l p}(Y, X)$ for every Banach space $Y$.

Theorem 2.3. If $X^{*}$ has the limited $p$-Schur property and $Y$ has the Schur property, then $L(X, Y)$ has the limited p-Schur property.

Proof. Since $X^{*}$ has the limited $p$-Schur property, Theorem 2.2 implies that each $\psi_{y^{*}}: L(X, Y) \rightarrow X^{*}$ is limited $p$-converging. It follows that $L(X, Y)$ has the limited $p$-Schur property. In fact, if $L(X, Y)$ does not have the limited $p$-Schur property, then there exists a limited weakly $p$-summable sequence $\left(T_{n}\right) \subseteq L(X, Y)$ such that $\left\|T_{n}\right\|>\varepsilon$ for all $n \in \mathbb{N}$ and some $\varepsilon>0$. Choose a sequence $x_{n} \in B_{X}$ such that $\left\|T_{n} x_{n}\right\|>\varepsilon$. On the other hand, $\psi_{y^{*}}$ is limited $p$-converging, for all $y^{*} \in Y^{*}$. Therefore $\left\|T_{n}^{*} y^{*}\right\|=\left\|\psi_{y^{*}} T_{n}\right\| \rightarrow 0$. It follows that

$$
\left|\left\langle T_{n} x_{n}, y^{*}\right\rangle\right| \leq\left\|T_{n}^{*} y^{*}\right\|\left\|x_{n}\right\| \rightarrow 0 .
$$

Hence $\left(T_{n} x_{n}\right)$ is weakly null and so is norm null. This contradiction shows that $L(X, Y)$ has the limited $p$-Schur property. 
Example 2.1. If $X^{*}$ has the limited $p$-Schur property, then $\ell_{1}^{\text {weak }}\left(X^{*}\right)$ has the same property. Indeed, if one denote $\ell_{1}^{\text {weak }}{ }^{*}\left(X^{*}\right)$ as the space of all sequences $\left(x_{n}^{*}\right) \subset X^{*}$ such that $\left(\left\langle x, x_{n}^{*}\right\rangle\right) \in \ell_{1}$, for all $x \in X$, then by $[5, P .427], \ell_{1}^{\text {weak }}\left(X^{*}\right)=\ell_{1}^{\text {weak }}{ }^{*}\left(X^{*}\right)$. Also, $\ell_{1}^{\text {weak }}{ }^{*}\left(X^{*}\right)$ is isometrically isomorphism to $L\left(X, \ell_{1}\right)$; see e.g., [8, Proposition 19.4.3]. Since $\ell_{1}$ has the Schur property, it follows that $L\left(X, \ell_{1}\right)=\ell_{1}^{\text {weak }}\left(X^{*}\right)$ has the limited $p$-Schur property.

If we take $p=\infty$ in Theorem 2.3 we obtain the following result.

Corollary 2.4. If $X^{*}$ has the GP property and $Y$ has the Schur property, then $L(X, Y)$ has the GP property.

Theorem 2.4. Let $X$ and $Y$ be Banach spaces. If $X$ has the limited $p$-Schur property and $Y$ has the GP property, then the space $K_{w^{*}}\left(X^{*}, Y\right)$ of all compact weak*-weak continuous operators from $X^{*}$ into $Y$ has the limited $p$-Schur property.

Proof. Let $\left(T_{n}\right)$ be a limited weakly $p$-summable sequence in $K_{w^{*}}\left(X^{*}, Y\right)$. We have to show that $\left\|T_{n}\right\| \rightarrow 0$. We can choose a sequence $\left(x_{n}^{*}\right)$ in $X^{*}$ such that $\left\|x_{n}^{*}\right\|=1$ and $\left\|T_{n} x_{n}^{*}\right\| \geq \frac{1}{2}\left\|T_{n}\right\|$ for all $n \in \mathbb{N}$. Now, we prove that $\left(T_{n} x_{n}^{*}\right)$ is weakly null limited sequence in $Y$. Fix any $y^{*} \in Y^{*}$. Then for all $T \in K_{w^{*}}\left(X^{*}, Y\right)$, the operator $y^{*} \circ T$ is a weak* continuous linear functional on $X^{*}$ so that $y^{*} \circ T \in X \subset X^{* *}$. Thus the operator $T \mapsto y^{*} \circ T$ from $K_{w^{*}}\left(X^{*}, Y\right)$ into $X$ shows that the sequence $\left(y^{*} \circ T_{n}\right)$ is limited weakly $p$-summable in $X$. So $\left\|y^{*} \circ T_{n}\right\| \rightarrow 0$ and for each $y^{*} \in Y^{*}$ we have

$$
\left\langle y^{*}, T_{n} x_{n}^{*}\right\rangle=\left\langle y^{*} \circ T_{n}, x_{n}^{*}\right\rangle \rightarrow 0
$$

and so $\left(T_{n} x_{n}^{*}\right)$ is weakly null.

Now, assume that $\left(y_{n}^{*}\right)$ is a weak*-null sequence in $Y^{*}$ and define a sequence $\left(\Lambda_{n}\right)$ in $K_{w^{*}}\left(X^{*}, Y\right)^{*}$ by $\left\langle T, \Lambda_{n}\right\rangle=\left\langle T x_{n}^{*}, y_{n}^{*}\right\rangle$. If $T \in K_{w^{*}}\left(X^{*}, Y\right)$, then $T\left(B_{X^{*}}\right)$ is relatively compact and so it is a limited set in $Y$. It follows that

$$
\lim _{n \rightarrow \infty} \sup _{x^{*} \in B_{X^{*}}}\left\langle T x^{*}, y_{n}^{*}\right\rangle=0 .
$$

Therefore $\left\|y_{n}^{*} \circ T\right\| \rightarrow 0$. Thus $\left\langle y_{n}^{*} \circ T, x_{n}^{*}\right\rangle \rightarrow 0$ and so $\left(\Lambda_{n}\right)$ is weak ${ }^{*}$-null in $K_{w^{*}}\left(X^{*}, Y\right)^{*}$. Since $\left(T_{n}\right)$ is limited, we have

$$
\left\langle T_{n} x_{n}^{*}, y_{n}^{*}\right\rangle=\left\langle T_{n}, \Lambda_{n}\right\rangle \rightarrow 0
$$

and so $\left(T_{n} x_{n}^{*}\right)$ is limited. Finally, the $G P$ property of $Y$ yields that $\left\|T_{n} x_{n}^{*}\right\| \rightarrow 0$ which implies $\left\|T_{n}\right\| \rightarrow 0$.

Note that the map $T \mapsto T^{* *}$ is an isometric isomorphism from $K(X, Y)$ into $K_{w^{*}}\left(X^{*}, Y\right)$. Therefore we have the following result.

Corollary 2.5. Let $X$ and $Y$ be two Banach spaces. If $X^{*}$ has the limited $p$-Schur property and $Y$ has the GP property, then $K(X, Y)$ has the limited $p$-Schur property. 
Since $X \widehat{\otimes}_{\varepsilon} Y$ may be identified with a closed subspace of $K_{w^{*}}\left(X^{*}, Y\right)$ via the isometric embedding $X \widehat{\otimes}_{\varepsilon} Y \hookrightarrow K_{w^{*}}\left(X^{*}, Y\right)$ which is defined by $x \otimes y \mapsto \theta_{x \otimes y}$, where $\theta_{x \otimes y}\left(x^{*}\right)=\left\langle x, x^{*}\right\rangle y$, we have the following corollary.

Corollary 2.6. If $X$ has the limited $p$-Schur property and $Y$ has the GP property, then injective tensor product $X \widehat{\otimes}_{\varepsilon} Y$ has the limited $p$-Schur property.

Theorem 2.5. [9,13] Let $X$ and $Y$ be two Banach spaces and $M \subseteq K(X, Y)$ such that for all $x \in X$, $M(x):=\{T x: T \in M\}$ is relatively compact in $Y$. Then under each of the following conditions, $M$ is a relatively compact subset of $K(X, Y)$.

(a) $X^{* *}$ has the GP property and for every weak ${ }^{*}$-null sequence $\left(x_{n}^{* *}\right) \subseteq X^{* *},\left(T^{* *} x_{n}^{* *}\right)$ is norm null uniformly with respect $T \in M$.

(b) $X$ contains no copy of $\ell_{1}$ and for every weakly null sequence $\left(x_{n}\right) \subseteq X,\left(T x_{n}\right)$ is norm null uniformly with respect $T \in M$.

Recall that the operator $T \in L(X, Y)$ is said to be limited operator if $T\left(B_{X}\right)$ is a limited set in $Y$. The class of all limited operator from $X$ into $Y$ is denoted by $L(X, Y)$. On the other hand, $T \in L(X, Y)$ if and only if $T^{*}: Y^{*} \rightarrow X^{*}$ is weak*-norm sequential continuous cf. [2].

Theorem 2.6. Let $X$ be a Banach space such that $X^{*}$ has the GP property. If $F$ is a closed subspace of $K(X, Y)$ and for every $x^{* *} \in X^{* *}$, the evaluation operator $\phi_{x^{* *}}$ on $F$ is limited $p$-converging, then $F$ has the limited p-Schur property.

Proof. First, observe that the evaluation operator $\phi_{x^{* *}}$, as a generalization of $\phi_{x}$ is denoted by $\phi_{x^{* *}}(T)=$ $T^{* *} x^{* *}$, for all $T \in M$ and $x^{* *} \in X^{* *}$.

Let $M \subset F$ be a limited weakly $p$-compact set. Since for every $x \in X$, the evaluation map $\phi_{x}$ is limited $p$ converging, we conclude that $M(x)=\{T x: T \in M\}$ is relatively compact. Since the adjoint of every limited operator is weak*-norm sequentially continuous, it follows that for every compact operator $T \in K(X, Y)$, the operator $T^{*}$ is also compact and so is limited. This shows that $T^{* *}$ is weak ${ }^{*}$-norm sequentially continuous and therefore for each weak*-null sequence $\left(x_{n}^{* *}\right)$ in $X^{* *}$, the sequence $\left(T^{* *} x_{n}^{* *}\right)$ is norm null, that is $\phi_{x^{* *}}$ is a pointwise norm null sequence of bounded linear operators. Hence $\left(\phi_{x_{n}^{* *}}\right)$ converges uniformly on the limited set $M[17$, Corollary 1.1.2]. It follows that

$$
\lim _{n \rightarrow \infty} \sup _{T \in M}\left\|\phi_{x_{n}^{* *}}(T)\right\|=0
$$

Then by Theorem 2.5 (a) $M$ is relatively compact and so $F$ has the $p$-Schur property.

If one use Theorem 2.5 (b) instead of Theorem 2.5 (a), we can prove the following theorem. 
Theorem 2.7. Let $X$ be a Banach space containing no copy of $\ell_{1}$. If $F$ is a closed subspace of $K(X, Y)$ such that for each $x \in X$, the evaluation operator $\phi_{x}$ is limited $p$-converging, then $F$ has the limited $p$-Schur property.

Recall that a subset $H$ of $L(X, Y)$ is uniformly completely continuous, if for every weakly null sequence $\left(x_{n}\right)$ in $X$,

$$
\lim _{n \rightarrow \infty} \sup _{T \in H}\left\|T x_{n}\right\|=0 .
$$

We remember the following theorem, which has a main role in the proof of the Theorem 2.9.

Theorem 2.8. [13] If $X$ contains no copy of $\ell_{1}$, then a subset $H \subseteq K(X, Y)$ is relatively compact if and only if $H$ is uniformly completely continuous and for each $x \in X$, the set $\phi_{x}(H)$ is relatively compact in $Y$.

Theorem 2.9. If $X$ contains no copy of $\ell_{1}$ and $Y$ has the limited $p$-Schur property, then $K(X, Y)$ has the limited p-Schur property.

Proof. If $Y$ has the limited $p$-Schur property, then Theorem 2.2 shows that each $\phi_{x}: K(X, Y) \rightarrow Y$ is limited $p$-converging. Now, suppose that $H \subset K(X, Y)$ is a limited weakly $p$-compact set. Therefore $\phi_{x}(H)$ is relatively compact for all $x \in X$. On the other hand, if $\left(x_{n}\right)$ is weakly null in $X$, then complete continuity of each operator $T \in H$ implies that $\left\|\phi_{x_{n}}(T)\right\|=\left\|T x_{n}\right\| \rightarrow 0$. Therefore $\left(\phi_{x_{n}}\right)$ is a norm null sequence at each element $T \in H$ and then it is uniformly convergent on the limited set $H$ [17, Corolarry 1.1.2]. Hence

$$
\lim _{n \rightarrow \infty} \sup _{T \in H}\left\|T x_{n}\right\|=\lim _{n \rightarrow \infty} \sup _{T \in H}\left\|\phi_{x_{n}}(T)\right\|=0 .
$$

This shows that $H$ is uniformly completely continuous. Hence Theorem 2.5 (a) shows that $H$ is relatively compact in $K(X, Y)$ and so $K(X, Y)$ has the limited $p$-Schur property.

Recall that if $1 \leq p \leq \infty$, the Banach space $X$ has the Dunford-Pettis property of order $\mathrm{p}\left(D P_{p}\right)$ if for each Banach space $\mathrm{Y}$, every weakly compact operator $T: X \rightarrow Y$ is $p$-converging. For more information about $D P_{p}$ property of Banach spaces the reader is referred to [3].

Corollary 2.7. If $2<q<\infty$ and $\frac{1}{q}+\frac{1}{q^{*}}=1$, then $\left(\ell_{q} \widehat{\otimes}_{\varepsilon} \ell_{q}\right)^{*}$ and $\left(\ell_{q} \widehat{\otimes}_{\pi} \ell_{q}\right)^{*}$ have the limited p-Schur property, for all $1<p<q$.

Proof. Since $1<q^{*}<2$ and $q^{*}<q<\infty$, by Pitt's Theorem; (see [1, Theorem 2.1.4]), every bounded operator $T: \ell_{q} \rightarrow \ell_{q^{*}}$ is compact. Therefore $\left(\ell_{q} \widehat{\otimes}_{\pi} \ell_{q}\right)^{*}=L\left(\ell_{q}, \ell_{q^{*}}\right)=K\left(\ell_{q}, \ell_{q^{*}}\right)$ and $\left(\ell_{q} \widehat{\otimes}_{\varepsilon} \ell_{q}\right)^{*}=I\left(\ell_{q}, \ell_{q^{*}}\right) \subset$ $K\left(\ell_{q}, \ell_{q^{*}}\right)$, where $I\left(\ell_{q}, \ell_{q^{*}}\right)$ is the space of all integral operators from $\ell_{q}$ into $\ell_{q^{*}}$ [5, P. 119]. Hence it is enough to show that $K\left(\ell_{q}, \ell_{q^{*}}\right)$ has the limited $p$-Schur property, for all $1<p<q$. In fact, by [3, Example 3.3] $\ell_{q^{*}}$ has the $D P_{p}$ property, for all $1<p<q$. It follows from [6, Theorem 2.31] that $\ell_{q^{*}}$ has the (limited) 
$p$-Schur property, for all $1<p<q$. On the other hand, $\ell_{q}$ contains no copy of $\ell_{1}$. Therefore Theorem 2.9 (or Corollary 2.5) shows that $K\left(\ell_{q}, \ell_{q^{*}}\right)$ has the limited $p$-Schur property, for all $1<p<q$.

We also notice that by Theorem 2.2, if the closed subspace $M$ of $L(X, Y)$ has the limited $p$-Schur property, then all operators on $M$, such as evaluation operators, are limited $p$-converging. Therefore the converse of Theorem 2.6 is also true. Moreover, in the following two theorems 2.11 and 2.12, we will give another sufficient conditions for the limited $p$-Schur property of closed subspace $M$ of some operator spaces with respect to the limited $p$-converging of evaluation operators.

To obtain our next result we need the following well known theorem.

Theorem 2.10. [9] Let $X$ and $Y$ be two Banach spaces and $H$ be a subset of $L(X, Y)$ such that

(1) $H\left(B_{X}\right)=\left\{T x: T \in H, x \in B_{X}\right\}$ is relatively compact.

(2) $\psi_{y^{*}}(H)$ is relatively compact for all $y^{*} \in Y^{*}$.

Then $H$ is relatively compact.

Theorem 2.11. Let $M$ be a closed linear subspace of $L(X, Y)$ such that the closed linear span of the set $M(X)=\{T x: T \in M, x \in X\}$ has the GP property. If all evaluation operator $\psi_{y^{*}}$ are limited $p$-converging, then $M$ has the limited $p$-Schur property.

Proof. Suppose that $H$ is a limited weakly $p$-compact subset of $M$. By Theorem 2.10 , it is enough to show that $H\left(B_{X}\right)$ and all $\psi_{y^{*}}(H)$ are relatively compact in $Y$ and $X^{*}$, respectively. For every $y^{*} \in Y^{*}$, the evaluation operator $\psi_{y^{*}}$ is limited $p$-converging. Therefore $\psi_{y^{*}}(H)$ is relatively compact. On the other hand, if $\left(y_{n}^{*}\right)$ is a weak*-null sequence in $Y^{*}$, then the weak*-norm sequential continuity of the adjoint of eah $T \in H$ implies that $\left\|\psi_{y_{n}^{*}}(T)\right\|=\left\|T^{*} y_{n}^{*}\right\| \rightarrow 0$ as $n \rightarrow \infty$. Therefore $\left(\psi_{y_{n}^{*}}\right)$ converges pointwise on $H$ an so it is converges uniformly on the subset $H$ of $M$. Hence

$$
\begin{aligned}
\sup \left\{\left|\left\langle T x, y_{n}^{*}\right\rangle\right|: T \in H, x \in B_{X}\right\} & =\sup \left\{\left|\left\langle x, T^{*} y_{n}^{*}\right\rangle\right|: T \in H, x \in B_{X}\right\} \\
& =\sup _{T \in H}\left\|T^{*} y_{n}^{*}\right\| \rightarrow 0 .
\end{aligned}
$$

Thus $H\left(B_{X}\right)$ is limited and so is relatively compact.

Now, we give a sufficient condition for the limited $p$-Schur property of subspaces of $L_{w^{*}}\left(X^{*}, Y\right)$ of all bounded weak ${ }^{*}$-weak continuous operator from $X^{*}$ to $Y$. Clearly, if $T \in L_{w^{*}}\left(X^{*}, Y\right)$, then $T^{*}$ transfers $Y^{*}$ into $X$. The proof of this theorem is similar to the proof of Theorem 3.6 of [6]. So we omit its proof.

Theorem 2.12. Let $X$ and $Y$ be Banach spaces such that $X$ has the Schur property. If $M$ is a closed subspace of $L_{w^{*}}\left(X^{*}, Y\right)$ such that every evaluation operator $\phi_{x^{*}}$ is limited $p$-converging on $M$, then $M$ has the limited $p$-Schur property. 
Recall that according to [6], a bounded subset $K$ of a Banach space $X$ is $p$-Limited if

$$
\lim _{n} \sup _{x \in K}\left|\left\langle x, x_{n}^{*}\right\rangle\right|=0
$$

for every $\left(x_{n}^{*}\right) \in \ell_{p}^{w e a k}\left(X^{*}\right)$.

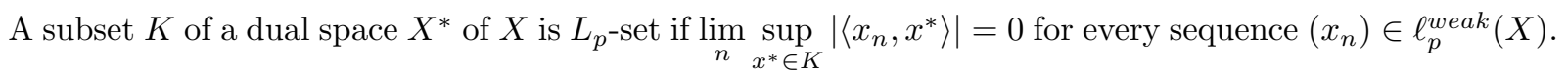
Also, a sequence $\left(x_{n}^{*}\right)$ in $X^{*}$ is an $L_{p}$-set if and only if $\lim _{n \rightarrow \infty}\left\langle x_{n}, x_{n}^{*}\right\rangle=0$ for all $\left(x_{n}\right) \in \ell_{p}^{\text {weak}}(X)$ [7]. It is clear that for every limited subset and every $p$-limited subset of a dual space is an $L_{p}$-set. Moreover, the following result has been proved in [7].

Theorem 2.13. A Banach space $X$ is weakly p-compact if and only if every $L_{p}$-set in $X^{*}$ is relatively compact.

Theorem 2.14. Let $X$ and $Y$ be Banach spaces. If $X$ contains no copy of $\ell_{1}, Y^{*}$ is weakly p-compact and for every $h \in L\left(X, Y^{* *}\right)$, for every weakly null sequence $\left(x_{n}\right) \subset X$, the sequence $\left(h x_{n}\right)$ is an $L_{p^{-}}$set, then $K(X, Y)$ has the GP property and so has the limited p-Schur property.

Proof. Let $M \subset K(X, Y)$ be a limited set. We have to prove that $M$ is relatively compact. Since $M(x)=$ $\{T x: T \in M\}$ is a limited set in $Y$ and so is an $L_{p}$-set, therefore $M(x)$ is a relatively compact set, by Theorem 2.13. Assume that condition (b) of Theorem 2.5 in not verified. So there are a positive number $\varepsilon$, a weakly null sequence $\left(x_{n}\right) \subset X$ and a sequence $\left(T_{n}\right) \subset M$ such that for all $n \in \mathbb{N},\left\|T_{n} x_{n}\right\|>\varepsilon$. Now we prove that $\left(T_{n} x_{n}\right)$ is weakly null. For every $y^{*} \in Y^{*}$, the set $\left\{T_{n}^{*} y^{*}: n \in \mathbb{N}\right\}$ is a Dunford-Pettis subset of $X^{*}$. Since $\left(x_{n}\right)$ is weakly null, it follows that

$$
\left\langle T_{n} x_{n}, y^{*}\right\rangle=\left\langle T_{n}^{*} y^{*}, x_{n}\right\rangle \rightarrow 0
$$

for every $y^{*} \in Y^{*}$. So the sequence $\left(T_{n} x_{n}\right)$ is weakly null.

Now, we prove that $\left(T_{n} x_{n}\right)$ is a $p$-limited set. Suppose that $\left(y_{n}^{*}\right) \in \ell_{p}^{w e a k}\left(Y^{*}\right)$ and $h \in\left(X \widehat{\otimes}_{\pi} Y^{*}\right)^{*}=$ $L\left(X, Y^{* *}\right)$. As $\left(h x_{n}\right)$ is an $L_{p^{-}}$set in $Y^{* *}$ we have $h\left(x_{n} \otimes y_{n}^{*}\right)=\left\langle h x_{n}, y_{n}^{*}\right\rangle \rightarrow 0$ and so $\left(x_{n} \otimes y_{n}^{*}\right)$ is weakly null in $X \otimes_{\pi} Y^{*}$. Since $X \widehat{\otimes}_{\pi} Y^{*}$ embeds into $K(X, Y)^{*}$, it follows that $\left(x_{n} \otimes y_{n}^{*}\right)$ is also weakly null in space $K(X, Y)^{*}$. Then it must be that

$$
\lim _{n \rightarrow \infty}\left\langle T_{n} x_{n}, y_{n}^{*}\right\rangle=\lim _{n \rightarrow \infty}\left\langle T_{n}, x_{n} \otimes_{\pi} y_{n}^{*}\right\rangle=0
$$

because $\left(T_{n}\right)$ is a limited set and so is a $D P$ set. So we have actually proved that $\left(T_{n} x_{n}\right)$ is a $p$-limited set and so $L_{p}$-set. It follows from Theorem 2.13 that it must be a relatively compact set. Since it is a weakly null sequence, there is a norm null subsequence and it is a contradiction.

In [18] the authors have been proved that for Banach spaces $\left(X_{\alpha}\right)_{\alpha \in I}$, if $X=\left(\oplus_{\alpha \in I} X_{\alpha}\right)_{1}$ is their $\ell_{1}$-direct sum, then $X$ has the Schur property if and only if each factor $X_{\alpha}$ has the same property. Here, by a similar idea, we prove that the same condition holds for (limited) $p$-Schur property. 
Theorem 2.15. Let $\left(X_{\alpha}\right)_{\alpha \in I}$ be Banach spaces and $X=\left(\oplus_{\alpha \in I} X_{\alpha}\right)_{1}$. Then the space $X$ has the $p$-Schur property if and only if each $X_{\alpha}$ has the $p$-Schur property.

Proof. If $X=\left(\oplus_{\alpha \in I} X_{\alpha}\right)_{1}$ has the $p$-Schur property, then clearly, every closed subspace of $X$ has the $p$-Schur property. Hence each $X_{\alpha}$ has the $p$-Schur property. On the other hand, a straightforward computations shows that a Banach space has the $p$-Schur property if and only if all of its closed separable subspaces have the $p$-Schur property. Therefore we can assume that each $X_{\alpha}$ is separable and take $I=\mathbb{N}$. Hence $X=\left(\oplus X_{k}\right)_{1}$ is separable and so has the GP property.

If $\left(x_{n}\right) \in \ell_{p}^{\text {weak }}(X)$, where $x_{n}=\left(b_{n, k}\right)_{k \in \mathbb{N}}$, then $\left(b_{n, k}\right) \in \ell_{p}^{\text {weak }}\left(X_{k}\right)$ for all $k \in \mathbb{N}$. Since $X_{k}$ has the $p$-Schur property, therefore $\left\|b_{n, k}\right\| \rightarrow 0$ as $n \rightarrow \infty$, for all $k \in \mathbb{N}$. We have to prove that $\left\|x_{n}\right\| \rightarrow 0$ or the weakly null sequence $\left(x_{n}\right)$ is relatively compact. Let $\left\{f_{n}\right\}_{n \in \mathbb{N}}$ be a $w^{*}$-null sequence in $B_{X^{*}}$. If we show that $\lim _{n \rightarrow \infty}\left\langle x_{n}, f_{n}\right\rangle=0$, then the proof is completed, thanks to the GP property of $X$.

Each $f_{n}$ is of the form $f_{n}=\left(a_{n, k}\right)_{k \in \mathbb{N}}$ and for all $k \in \mathbb{N}, a_{n, k} \stackrel{w^{*}}{\longrightarrow} 0$ in $X_{k}^{*}$ as $n \rightarrow \infty$. To prove that $\lim _{n \rightarrow \infty}\left\langle x_{n}, f_{n}\right\rangle=0$, it is enough to show that

$$
\sup _{n} \sum_{k>M}\left|\left\langle a_{n, k}, b_{n, k}\right\rangle\right| \rightarrow 0 \text { as } M \rightarrow \infty .
$$

Therefore we have to show that for each $\varepsilon>0$ there exists $M \in \mathbb{N}$ such that

$$
\sum_{k>M}\left|\left\langle a_{n, k}, b_{n, k}\right\rangle\right|<\varepsilon
$$

for all sufficiently large enough $n \in \mathbb{N}$. Let (2.1) is false. Then there is an $\varepsilon>0$ such that

$$
\sum_{k>M}\left|\left\langle a_{n, k}, b_{n, k}\right\rangle\right| \geq \varepsilon
$$

for all $M \in \mathbb{N}$ and some sufficiently large enough $n \in \mathbb{N}$. Consider a sequence of positive number, $\left(\delta_{k}\right)$ such that $\sum_{k=1}^{\infty} \delta_{k}<\frac{\varepsilon}{4}$. By the technique given in the proof of main theorem of [18] one can construct two strictly increasing sequences, $\left(n_{k}\right)_{k \geq 1}$ and $\left(M_{k}\right)_{k \geq 0}$ such that

(1) $\sum_{j>M_{k}}\left\|b_{n_{k}, j}\right\| \leq \delta_{k}$ for each $k \geq 1$

(2) $\sum_{j=1}^{M_{k-1}}\left|\left\langle a_{n, j}, b_{n_{k-1}}, j\right\rangle\right| \leq \delta_{k}$ for each $n \geq n_{k}$

(3) $\sum_{j>M_{k-1}}\left|\left\langle a_{n_{k}, j}, b_{n_{k}}, j\right\rangle\right| \geq \varepsilon$.

Now, let us choose a sequence $\left(\lambda_{j}\right)$ such that $\left|\lambda_{j}\right|=1$, for all $j$ and

$$
\lambda_{j}\left\langle a_{n_{k}, j}, b_{n_{k}, j}\right\rangle=\left|\left\langle a_{n_{k}, j}, b_{n_{k}, j}\right\rangle\right|,
$$

where $k \geq 1$ and $M_{k-1}+1 \leq j \leq M_{k}$. Let

$$
h=\left(h_{j}\right)_{j \geq 1}=\left(\lambda_{1} a_{n_{1}, 1}, \lambda_{2} a_{n_{1}, 2}, \ldots, \lambda_{M_{1}} a_{n_{1}, M_{1}}, \lambda_{M_{1}+1} a_{n_{2}, M_{1}+1}, \ldots\right) .
$$


Then $\|h\|=\sup _{j \geq 1}\left\|h_{j}\right\| \leq 1$ and

$$
\begin{array}{r}
\left\langle h, x_{n_{k}}\right\rangle=\sum_{j=1}^{\infty}\left\langle h_{j}, b_{n_{k}, j}\right\rangle=\sum_{i=1}^{k-1} \sum_{j=M_{i-1}+1}^{M_{i}} \lambda_{j}\left\langle a_{n_{i}, j}, b_{n_{i}, j}\right\rangle \\
+\sum_{j=M_{k-1}+1}^{M_{k}}\left|\left\langle a_{n_{k}, j}, b_{n_{k}, j}\right\rangle\right|+\sum_{j=M_{k}}^{\infty} \lambda_{j}\left\langle a_{n_{k}, j}, b_{n_{k}, j}\right\rangle .
\end{array}
$$

with due attention to $\left\|a_{n_{k}, j}\right\| \leq 1$ and inequalities (1), (2) and (3):

$$
\begin{aligned}
\left|\left\langle h, x_{n_{k}}\right\rangle\right| & \geq-\sum_{i=1}^{k-1} \delta_{i}+\sum_{j=M_{k-1}+1}^{M_{k}}\left|\left\langle a_{n_{k}, j}, b_{n_{k}, j}\right\rangle\right|-\delta_{k} \\
& \geq-\sum_{i=1}^{k-1} \delta_{i}+\sum_{j>M_{k-1}}\left|\left\langle a_{n_{k}, j}, b_{n_{k}, j}\right\rangle\right|-\sum_{j \leq M_{k-1}}\left|\left\langle a_{n_{k}, j}, b_{n_{k}, j}\right\rangle\right|-\delta_{k} \\
& \geq-\sum_{i=1}^{k-1} \delta_{i}+\sum_{j>M_{k-1}}\left|\left\langle a_{n_{k}, j}, b_{n_{k}, j}\right\rangle\right|-2 \delta_{k} \\
& \geq \varepsilon-\sum_{i=1}^{k-1} \delta_{i}-2 \delta_{k} \geq \varepsilon-2 \sum_{i=1}^{\infty} \delta_{i}>\frac{\varepsilon}{2} .
\end{aligned}
$$

This contradiction shows that (2.1) is true. So

$$
\lim _{M \rightarrow \infty} \sup _{n \in \mathbb{N}} \sum_{k=1}^{M}\left|\left\langle a_{n, k}, b_{n, k}\right\rangle\right|=0
$$

Therefore $\lim _{n \rightarrow \infty} \sum_{k=1}^{\infty}\left|\left\langle a_{n, k}, b_{n, k}\right\rangle\right|=\sum_{k=1}^{\infty} \lim _{n \rightarrow \infty}\left|\left\langle a_{n, k}, b_{n, k}\right\rangle\right|=0$ Since $\left|\left\langle f_{n}, x_{n}\right\rangle\right| \leq \sum_{k=1}^{\infty}\left|\left\langle a_{n, k}, b_{n, k}\right\rangle\right|$ we conclude that $\lim _{n \rightarrow \infty}\left|\left\langle f_{n}, x_{n}\right\rangle\right|=0$ and so $\left\|x_{n}\right\| \rightarrow 0$.

By a similar technique we have the following theorem.

Theorem 2.16. Suppose that $\left(X_{\alpha}\right)_{\alpha \in I}$ are Banach spaces and $X=\left(\oplus_{\alpha \in I} X_{\alpha}\right)_{1}$. Then the space $X$ has the limited p-Schur property if and only if each $X_{\alpha}$ has the limited p-Schur property.

\section{REFERENCES}

[1] F. Albiac and N.J. Kalton, Topics in Banach Space Theory, Graduate Texts in Mathematics, 233, Springer, New York, 2006.

[2] J. Bourgain and J. Diestel, Limited operators and strict consingularity, Math. Nachr. 119 (1984) 55-58.

[3] J. Castillo and F. Sanchez, Dunford-Pettis-like properties of continuous vector function spaces, Rev. Mat. Univ. Complut. Madrid 6 (1993), no. 1, 43-59.

[4] D. Chen, J. Alejandro Chvez-Domnguez, and Li. Lei. Unconditionally p-converging operators and Dunford-Pettis Property of order $p$, arXiv preprint arXiv:1607.02161 (2016).

[5] A. Defant and K. Floret, Tensor Norms and Operator Ideals, North-Holland Mathematics Studies, 176, North-Holland Publishing Co., Amsterdam, 1993.

[6] Mohammad B. Dehghani and S. Mohammad Moshtaghioun, On the p-Schur property of Banach spaces, Ann. Funct. Anal. (2017), 14 pages. 
[7] M.B. Dehghani and S.M. Moshtaghioun, Limited p-converging operators and its relation with some geometric properties of Banach spaces, (2017), Submitted.

[8] J. Diestel, H. Jachowr and A. Tonge, Absolutely summing operators, Cambrigde University Press, 1995.

[9] G. Emmanuele, On relative compactness in $K(X, Y)$, J. Math. Anal. Appl. 379 (2013) 88-90.

[10] J. H. Fouire and E. D. Zeekoei, DP* properties of order p on Banach spaces, Quaest. Math. 37 (2014), no. 3, 349-358.

[11] I. Ghencia and P. Lewis, The Dunford-Pettis property, the Gelfand-Phillips property and L-set, Colloq. Math. 1.6 (2006), 311-324.

[12] H. Jarchow, Locally convex spaces, B.G. Teubner, 1981.

[13] F. Mayoral, Compact sets of compact operators in absence of $\ell_{1}$, Proc. Amer. Math. Soc. 129 (2001), 7982.

[14] S.M. Moshtaghioun and J. Zafarani, Weak sequentional convergence in the dual of operator ideas, J. Oper. Theory 49 (2003), 143-151.

[15] A. Pelczynski, Banach Spaces in which every unconditionally converging operator is weakly compact, Bull. L'Acad. Polon. Sci. 10 no. 2, (1962), 641-648.

[16] R. Ryan, The Dunford-Pettis property and projective tensor products, Bull. Polish Acad. Sci. 35 no. 11-12, (1987), 785-792.

[17] T. Schlumprecht, Limited sets in Banach spaces, Ph. D. Dissertation, München, (1987).

[18] B. Tanbay, Direct sums and the Schur property, Turk. J. Math. 22 (1999), 349-354. 